\title{
Chemotherapy in Premenopausal Breast Cancer Patients
}

\author{
Ann H. Partridge \\ Dana-Farber Cancer Institute, Harvard Medical School, Boston, MA, USA
}

\section{Keywords \\ Breast Cancer - Adjuvant chemotherapy . \\ Premenopausal women · Long-term effects}

\section{Summary}

Evidence has long demonstrated that premenopausal women obtain the greatest benefit from adjuvant chemotherapy overall, with risk reduction increasing with decreasing age. The chemoendocrine effect of chemotherapy has only more recently been documented as impacting on outcomes for women with hormone receptor-positive breast cancer, and recent data have elucidated the optimal strategies for manipulating the menopausal status to improve disease outcomes, without necessarily including cytotoxic chemotherapy. Still, many premenopausal women will require adjuvant cytotoxic chemotherapy, and the effects of treatment on women diagnosed with breast cancer in the premenopausal setting can have important implications both on their breast cancer outcomes and on comorbidities and psychosocial outcomes. This article describes the most recent information and issues surrounding the indications, effects, and special considerations for adjuvant chemotherapy in premenopausal women with breast cancer, in an effort to inform their care.

(C) 2015 S. Karger GmbH, Freiburg

\section{Introduction}

Menopausal status is an important consideration when caring for women with breast cancer. Among premenopausal women, manipulation of ovarian functioning in the treatment of hormone receptor (HR)-positive disease has been a mainstay of therapy for over a century [1]. Evidence has also long demonstrated that premenopausal women obtain the greatest benefit from adjuvant chemotherapy overall, with risk reduction increasing with decreasing age [2]. However, the chemoendocrine effect of chemotherapy has only more recently been documented as impacting on outcomes for women with HR-positive breast cancer and recent data have elucidated the optimal strategies for manipulation of the menopausal status to improve disease outcomes, without necessarily including cytotoxic chemotherapy [3-6]. Nevertheless, many premenopausal women will require adjuvant cytotoxic chemotherapy, and the effects of breast cancer treatment on premenopausal women can profoundly impact not only on breast cancer outcomes but also on comorbidities and psychosocial outcomes [7, 8]. This article describes the most recent information and issues surrounding the indications, effects, and special considerations for adjuvant chemotherapy in premenopausal women with breast cancer, in an effort to inform their care.

\section{Indications for Adjuvant Chemotherapy}

Premenopausal women, and especially very young women (i.e., $<35$ years of age), are more likely to present with more advanced disease than older women $[9,10]$. They are also more likely to develop more aggressive subtypes of breast cancer with unfavorable prognostic features, and are less responsive to conventional therapy compared with disease arising in older premenopausal or postmenopausal women [11-13]. Tumors in younger women are more likely to be of high grade and HR negative; they have a high proliferation fraction and show more lymphovascular invasion. Younger women are also more likely to have human epidermal growth factor receptor 2 (HER2)-positive disease, with the highest incidence in the youngest women $[13,14]$. For example, in the population-based California Cancer Registry data, Keegan et al. [13] reported the incidence of

\section{KARGER \\ Fax +497614520714

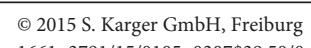

Ann H. Partridge, MD, MPH 
triple-negative breast cancer at 23\% for women 15-39 years of age and at $14 \%$ for women $40-49$ years of age, in contrast to $12 \%$ for women aged 50 years and older at diagnosis. The HER2-positive rates were $28 \%, 22 \%$, and $17 \%$ for the youngest to oldest age groups and there was a corresponding decreased incidence of luminal-type tumors as the women aged. Thus, in general, because of their higherrisk disease and associated greater risk of recurrence and mortality, and substantial improvements in disease outcomes with systemic chemotherapy and biologic therapy, the optimal treatment of premenopausal women often necessitates chemotherapy.

In recent years, as biologic subtyping has improved, it has been increasingly recognized that many premenopausal women have lower-risk HR-positive disease and can safely avoid chemotherapy with its associated long- and short-term toxicity, with good disease outcomes $[6,15-17]$. The international effort to study the value of chemotherapy in premenopausal women with early-stage chemotherapy, the Premenopausal Endocrine Responsive Chemotherapy (PERCHE) trial, which randomized women receiving combined endocrine therapy to chemotherapy or no chemotherapy, closed due to poor accrual. The TailoRx trial (www.ecog.dfci.harvard. $e d u$ ), a trial that includes both pre- and postmenopausal women and randomized those whose tumors have intermediate Oncotype Dx scores to chemotherapy or no chemotherapy, is fully accrued. A similar trial for node-positive women, the RxPonder trial ( $w w w$. swog.org), is recruiting. Collectively, these studies may provide additional information on the role of chemotherapy in premenopausal women with HR-positive breast cancer. Yet, prior data do support forgoing chemotherapy in some premenopausal patients. For example, the Austrian Breast Cancer Study Group 12 randomized over 1,800 premenopausal women with HR-positive stage 1 or 2 breast cancer, only $5 \%$ of whom had undergone any chemotherapy, to adjuvant ovarian suppression with either anastrozole or tamoxifen, with a second randomization to zoledronic acid or not for 3 years. At 62 months, differences between the groups were notable for improvements seen from adding zoledronic acid, but the overall disease-free and overall survival rates were in the range of 88 $92 \%$ and $95-97 \%$, respectively, considering all treatment groups $[16,17]$. Given that the benefits of adjuvant chemotherapy are demonstrated in the first 5 years after diagnosis [2], such high rates of freedom from disease at that time suggest that these women had not indeed needed chemotherapy, in general.

Thus, current recommendations advise that adjuvant treatment be based on disease and patient characteristics predicting the risk of systemic recurrence and potential responsiveness to therapy, as well as patient preferences and values [18]. Treatment recommendations should be tailored, regardless of menopausal status or age, to the phenotypic subtype of the tumor as assessed by conventional factors, such as estrogen receptor (ER), progesterone receptor (PR), and HER2 expression, as well as grade and proliferation rate. Genetic signature technology, when available, may provide additional predictive information and risk stratification regarding the degree of risk and responsiveness to therapy, although there are still only limited data available to guide the use of available tests in premenopausal women [19-21].

\section{Chemotherapy Selection for Premenopausal Women}

While the consideration of endocrine responsiveness should be the first consideration in tailoring adjuvant therapies for patients with breast cancer, regardless of menopausal status, adjuvant chemotherapy has been used extensively in premenopausal patients because of its overwhelming beneficial effects on outcome in certain patient subgroups [2]. As noted previously, premenopausal women are more likely to develop HER2-positive and triplenegative tumors compared with postmenopausal women, and the benefits of chemotherapy in these groups is well documented, regardless of menopausal status [22, 23]. Furthermore, women with luminal tumors appear to benefit from chemotherapy, although discerning for whom the benefits outweigh risks has been challenging [24].

For premenopausal women with HR-negative disease, adjuvant chemotherapy is a very important component of successful treatment. Studies have suggested that women with ER-negative disease garner the greatest benefits from incremental benefits from new chemotherapeutic strategies, and this does not appear to vary substantially with age or menopausal status. For example, women with ER-negative disease appeared to get the greatest benefit from the addition of paclitaxel to adriamycin and cytoxan and from giving the chemotherapy in a dose-dense fashion, in the Cancer and Leukemia Group B (CALGB) studies 9344 and 9741, respectively [25, 26]. One study has prospectively tested the use of chemotherapy in women with HR-negative, node-negative disease (National Surgical Adjuvant Breast and Bowel Project (NSABP) B13) [27]. Premenopausal women experienced a $38 \%$ reduction in the risk of recurrence from chemotherapy compared with surgery alone, and there was no difference between very young and older premenopausal women. Standard options for adjuvant chemotherapy for non-HER2-positive breast cancer are further detailed in current guidelines (www.NCCN.com)

Novel chemotherapeutic regimens (e.g., platinum agents) and biologics (e.g., poly(ADP-ribose) polymerase (PARP) inhibitors) that have shown promise in women with early and advanced disease may also be particularly relevant in the treatment of premenopausal women with ER-negative disease, given that this population is more likely to develop triple-negative disease and also more likely to harbor a BRCA1 or BRCA2 germline mutation [28-31].

The incremental benefits of the anti-HER2 therapy, trastuzumab, appear to be present across age groups, regardless of menopausal status $[22,23]$. In an analysis of the HERceptin Adjuvant (HERA) trial including women with early-stage HER2-positive breast cancer, age was not strongly associated with risk of early recurrence or prediction of benefit from trastuzumab therapy [32]. Standard adjuvant chemotherapy and biologic options for HER2-positive disease are outlined in current guidelines ( $w w w$. NCCN.com).

For premenopausal women with HR-positive disease, longstanding controversy has existed about optimal management. The Early Breast Cancer Trialists' Collaborative Group (EBCTCG) meta-analysis regarding ovarian ablation revealed that the benefi- 
cial effect of ovarian ablation was primarily seen only in the absence of chemotherapy, noting that more than $80 \%$ of the chemotherapy-alone group experiences amenorrhea with treatment [1, 33]. The International Breast Cancer Study Group (IBCSG) VIII study revealed that very young $(<35$ years old) women who received adjuvant chemotherapy alone for HR-positive tumors fared worse than older premenopausal women, leading to the recognition of and further validation studies on the chemoendocrine effect of chemotherapy in premenopausal women: Older premenopausal women are more likely to undergo chemotherapy-related ovarian dysfunction compared to young premenopausal women, resulting in improved disease outcomes in the setting of HR-positive breast cancer $[3,4,34,35]$. Thus, the beneficial effects of chemotherapy in premenopausal women with HR-positive breast cancer are likely a result of a combination of cytotoxic and endocrine effects of chemotherapy.

\section{Side Effects of Chemotherapy in Premenopausal Patients}

Short-term symptoms and long-term or late effects of adjuvant chemotherapy should be considered in decision-making, treating and, in the long-term, follow-up care of premenopausal women with early-stage breast cancer. In general, premenopausal women tolerate chemotherapy as well as older women $[8,36]$. However, the menopausal transition with therapy has been associated with both short- and long-term problems [7, 37]. Infertility, menopausal symptoms and sexual dysfunction, weight gain, body image concerns, and psychosocial distress are all common in premenopausal breast cancer survivors [38-40]. Research has, however, identified risk factors for sexual dysfunction in breast cancer survivors, including younger age, premature menopause, and the use of chemotherapy [41]. Fortunately, interventions to improve menopausal symptoms and sexual functioning have proven effective, with tailored specific attention focused on the problems and symptoms [42]. Guidelines to preserve fertility as well as psychosocial interventions exist to improve outcomes in patients with cancer and should be considered when caring for this vulnerable population (www.asco.org).

There are also numerous potential medical effects of premature menopause, including increased risks of weight gain, bone loss, cardiovascular disease, and dementia [43]. Attention to these issues, including prevention, screening and management, depending on the risk and the problem, in both short- and long-term followup is warranted (table 1).
Table 1. Special issues surrounding the treatment of premenopausal breast cancer patients with chemotherapy

- The benefits of chemotherapy treatment, particularly in light of potentia benefits of optimal endocrine therapy, should be weighed against the potential risks.

- Selection of the chemotherapy regimen should depend on disease risk and phenotype as well as on patient preferences and comorbidities.

- Particular attention to menopausal symptoms and potential long-term risks of premature menopause is necessary.

- Premenopausal patients have an increased risk of distress in long- and short-term follow-up, warranting screening and treatment as needed.

\section{Special Considerations}

Premenopausal women with breast cancer face a variety of problems unique to or accentuated by their menopausal status and potential for a transition with treatment, and young age. In particular, younger women are more likely to be diagnosed at a time in life when roles at home or work may be disrupted by treatment, particularly when it includes chemotherapy. Furthermore, beauty, body image, and fertility may be of substantial importance for the younger population, all of which can be impaired by treatment. These concerns may contribute to the greater psychosocial distress seen in younger women at both diagnosis and in follow-up [7]. Women who undergo a menopausal transition with treatment may be particularly at risk. In a cross-sectional survey of 577 women ranging in age from 30 to 62 years, approximately 6 years after diagnosis - where nearly $75 \%$ had received adjuvant therapy amenorrhea occurred frequently as a result of treatment in women $\geq 40$ years at diagnosis [37]. Treatment-associated menopause was associated with poorer health perceptions. While the overall quality of life (QOL) in premenopausal breast cancer survivors is generally good, there is clearly substantial symptomatology and risk of increased emotional disruption, especially among women experiencing a menopausal transition as part of the therapy [37, 44]. Thus, treating premenopausal women with adjuvant breast cancer chemotherapy warrants not only attention to the myriad shortand long-term side effects and medical risks but also to the potential for emotional distress, and screening and appropriate intervention for the psychosocial aftermath of treatment comprise an important component of high-quality cancer care [45].

\section{Disclosure Statement}

The author has no conflicts of interest to disclose. 


\section{References}

1 Goel S, Sharma R, Hamilton A, Beith J: LHRH agonists for adjuvant therapy of early breast cancer in premenopausal women. Cochrane database of systematic reviews 2009(4): CD004562.

2 Polychemotherapy for early breast cancer: an overview of the randomised trials. Early Breast Cancer Trialists Collaborative Group. Lancet 1998;352:930-942.

3 Swain SM, Jeong JH, Geyer CE Jr, et al.: Longer therapy, iatrogenic amenorrhea, and survival in early breast cancer. N Engl J Med 2010;362:2053-2065.

4 Pagani O, O’Neill A, Castiglione M, et al.: Prognostic impact of amenorrhoea after adjuvant chemotherapy in premenopausal breast cancer patients with axillary node involvement: results of the International Breast Cancer Study Group (IBCSG) Trial VI. Eur J Cancer 1998;34:632-640.

5 Pagani O, Regan MM, Walley BA, et al.: Adjuvant exemestane with ovarian suppression in premenopausal breast cancer. N Engl J Med 2014;371:107-118.

6 Francis PA, Regan MM, Fleming GF, et al.: Adjuvant ovarian suppression in premenopausal breast cancer. N Engl J Med 2015;372:436-446.

7 Howard-Anderson J, Ganz PA, Bower JE, Stanton AL: Quality of life, fertility concerns, and behavioral health outcomes in younger breast cancer survivors: a systematic review. J Natl Cancer Inst 2012;104:386-405.

8 Partridge AH, Burstein HJ, Winer EP: Side effects of chemotherapy and combined chemohormonal therapy in women with early-stage breast cancer. J Natl Cancer Inst Monogr 2001;135-142.

9 Partridge AH, Hughes ME, Ottesen RA, et al.: The effect of age on delay in diagnosis and stage of breast cancer. Oncologist 2012;17:775-782.

10 Warner ET, Tamimi RM, Hughes ME, et al.: Time to diagnosis and breast cancer stage by race/ethnicity. Breast Cancer Res Treat 2012;136:813-821.

11 Colleoni M, Rotmensz N, Peruzzotti G, et al.: Role of endocrine responsiveness and adjuvant therapy in very young women (below 35 years) with operable breast cancer and node negative disease. Ann Oncol 2006; 17 1497-1503.

12 Ahn SH, Son BH, Kim SW, et al.: Poor outcome of hormone receptor-positive breast cancer at very young age is due to tamoxifen resistance: nationwide survival data in Korea - a report from the Korean Breast Cancer Society. J Clin Oncol 2007;25:2360-2368.

13 Keegan TH, DeRouen MC, Press DJ, et al.: Occurrence of breast cancer subtypes in adolescent and young adult women. Breast Cancer Res 2012;14:R55.

14 Collins LC, Marotti JD, Gelber S, et al.: Pathologic features and molecular phenotype by patient age in a large cohort of young women with breast cancer. Breast Cancer Res Treat 2012;131:1061-1066.

15 Thurlimann B, Price KN, Gelber RD, et al.: Is chemotherapy necessary for premenopausal women with lower-risk node-positive, endocrine responsive breast cancer? 10-year update of International Breast Cancer Study Group Trial 11-93. Breast Cancer Res Treat 2009;113:137-144.

16 Gnant M, Mlineritsch B, Stoeger H, et al.: Adjuvant endocrine therapy plus zoledronic acid in premenopausal women with early-stage breast cancer: 62-month follow-up from the ABCSG-12 randomised trial. Lancet Oncol 2011;12:631-641.
17 Gnant M, Mlineritsch B, Schippinger W, et al.: Endocrine therapy plus zoledronic acid in premenopausal breast cancer. N Engl J Med 2009;360:679-691.

18 Coates AS, Winer EP, Goldhirsch A, et al.: Tailoring therapies - improving the management of early breast cancer: St Gallen International Expert Consensus on the Primary Therapy of Early Breast Cancer 2015. Ann Oncol 2015;26:1533-1546.

19 Paik S, Shak S, Tang G, et al.: A multigene assay to predict recurrence of tamoxifen-treated, node-negative breast cancer. N Engl J Med 2004;351:2817-2826.

20 Gnant M, Sestak I, Filipits M, et al.: Identifying clinically relevant prognostic subgroups of postmenopausal women with node-positive hormone receptor-positive early-stage breast cancer treated with endocrine therapy: a combined analysis of ABCSG-8 and ATAC using the PAM50 risk of recurrence score and intrinsic subtype. Ann Oncol 2015;26:1685-1691.

21 Hayes DF: Clinical utility of genetic signatures in selecting adjuvant treatment: risk stratification for early vs. late recurrences. Breast 2015, in press. DOI: 10.1016/j.breast.2015.07.002

22 Piccart-Gebhart MJ, Procter M, Leyland-Jones B, et al.: Trastuzumab after adjuvant chemotherapy in HER2positive breast cancer. N Engl J Med 2005;353:16591672.

23 Romond EH, Perez EA, Bryant J, et al.: Trastuzumab plus adjuvant chemotherapy for operable HER2-positive breast cancer. N Engl J Med 2005;353:1673-1684

24 Fisher B, Dignam J, Wolmark N, et al.: Tamoxifen and chemotherapy for lymph node-negative, estrogen receptor-positive breast cancer. J Natl Cancer Inst 1997; 89:1673-1682.

25 Henderson IC, Berry DA, Demetri GD, et al.: Improved outcomes from adding sequential paclitaxel but not from escalating doxorubicin dose in an adjuvant chemotherapy regimen for patients with node-positive primary breast cancer. J Clin Oncol 2003;21:976-983.

26 Citron ML, Berry DA, Cirrincione C, et al.: Randomized trial of dose-dense versus conventionally scheduled and sequential versus concurrent combination chemotherapy as postoperative adjuvant treatment of node-positive primary breast cancer: first report of Intergroup Trial C9741/Cancer and Leukemia Group B Trial 9741. J Clin Oncol 2003;21:1431-1439.

27 Fisher B, Dignam J, Mamounas EP, et al.: Sequential methotrexate and fluorouracil for the treatment of node-negative breast cancer patients with estrogen receptor-negative tumors: eight-year results from $\mathrm{Na}$ tional Surgical Adjuvant Breast and Bowel Project (NSABP) B-13 and first report of findings from NSABP B-19 comparing methotrexate and fluorouracil with conventional cyclophosphamide, methotrexate, and fluorouracil. J Clin Oncol 1996;14:1982-1992.

28 Kwon JS, Gutierrez-Barrera AM, Young D, et al.: Expanding the criteria for BRCA mutation testing in breast cancer survivors. J Clin Oncol 2010;28:42144220.

29 Guan X, Ma F, Fan Y, et al.: Platinum-based chemotherapy in triple-negative breast cancer: a systematic review and meta-analysis of randomized-controlled trials. Anticancer Drugs 2015;26:894-901.
30 Telli M: Evolving treatment strategies for triple-negative breast cancer. J Natl Compr Canc Netw 2015;13: 652-654.

31 Rios J, Puhalla S: PARP inhibitors in breast cancer BRCA and beyond. Oncology (Williston Park) 2011; 25:1014-1025

32 Partridge AH, Gelber S, Piccart-Gebhart MJ, et al.: Effect of age on breast cancer outcomes in women with human epidermal growth factor receptor 2-positive breast cancer: results from a herceptin adjuvant trial. J Clin Oncol 2013;31:2692-2698.

33 Bach PB, Mirkin JN, Oliver TK, et al.: Benefits and harms of CT screening for lung cancer: a systematic review. JAMA 2012;307:2418-2429.

34 Walshe JM, Denduluri N, Swain SM: Amenorrhea in premenopausal women after adjuvant chemotherapy for breast cancer. J Clin Oncol 2006;24:5769-5779.

35 International Breast Cancer Study Group (IBCSG), Castiglione-Gertsch M, O’Neill A, et al.: Adjuvant chemotherapy followed by goserelin versus either modality alone for premenopausal lymph node-negative breast cancer: a randomized trial. J Natl Cancer Inst 2003;95:1833-1846.

36 Partridge AH, Winer EP: Long-term complications of adjuvant chemotherapy for early stage breast cancer. Breast Dis 2004;21:55-64

37 Ganz PA, Greendale GA, Petersen L, et al.: Breast cancer in younger women: reproductive and late health effects of treatment. J Clin Oncol 2003;21:41844193.

38 Ruddy KJ, Partridge AH: Fertility (male and female) and menopause. J Clin Oncol 2012;30:3705-3711.

39 Ruddy KJ, Gelber SI, Tamimi RM, et al.: Prospective study of fertility concerns and preservation strategies in young women with breast cancer. J Clin Oncol 2014 32:1151-1156

40 Barton DL, Ganz PA: Symptoms: menopause, infertility, and sexual health. Adv Exp Med Biol 2015;862: 115-141.

41 Schover LR: Premature ovarian failure and its consequences: vasomotor symptoms, sexuality, and fertility. J Clin Oncol 2008;26:753-758.

42 Ganz PA, Greendale GA, Petersen L, et al.: Managing menopausal symptoms in breast cancer survivors: results of a randomized controlled trial. J Natl Cancer Inst 2000;92:1054-1064

43 Ewertz M, Jensen AB: Late effects of breast cancer treatment and potentials for rehabilitation. Acta Oncol 2011;50:187-193.

44 Ribi K, Luo W, Bernhard J, et al.: Patient-reported endocrine symptoms, sexual functioning and quality of life (QoL) in the IBCSG SOFT trial: adjuvant treatment with tamoxifen ( $\mathrm{T}$ ) alone versus $\mathrm{T}$ plus ovarian function suppression (OFS) in premenopausal women with hormone receptor-positive $(\mathrm{HR}+)$ early breast cancer (BC). San Antonio Breast Cancer Symposium 2015; abstr S3-09.

45 Partridge AH, Jacobsen PB, Andersen BL: Challenges to standardizing the care for adult cancer survivors: highlighting ASCO's fatigue and anxiety and depression guidelines. Am Soc Clin Oncol Educ Book 2015; 188-194. 